\title{
Finite-size corrections of the Entanglement Entropy of critical quantum chains
}

\author{
J. C. Xavier ${ }^{1,2}$ and F. C. Alcaraz ${ }^{2}$ \\ ${ }^{1}$ Instituto de Física, Universidade Federal de Uberlândia, \\ Caixa Postal 593, 38400-902 Uberlândia, MG, Brazil \\ ${ }^{2}$ Instituto de Física de São Carlos, Universidade de São Paulo, \\ Caixa Postal 369, 13560-970 São Carlos, SP, Brazil
}

(Dated: September 26, 2018)

\begin{abstract}
Using the density matrix renormalization group, we calculated the finite-size corrections of the entanglement $\alpha$-Rényi entropy of a single interval for several critical quantum chains. We considered models with $U(1)$ symmetry like the spin-1/2 XXZ and spin-1 Fateev-Zamolodchikov models, as well as models with discrete symmetries such as the Ising, the Blume-Capel, and the three-state Potts models. These corrections contain physically relevant information. Their amplitudes, that depend on the value of $\alpha$, are related to the dimensions of operators in the conformal field theory governing the long-distance correlations of the critical quantum chains. The obtained results together with earlier exact and numerical ones allow us to formulate some general conjectures about the operator responsible for the leading finite-size correction of the $\alpha$-Rényi entropies. We conjecture that the exponent of the leading finite-size correction of the $\alpha$-Rényi entropies is $p_{\alpha}=2 X_{\epsilon} / \alpha$ for $\alpha>1$ and $p_{1}=\nu$, where $X_{\epsilon}$ denotes the dimensions of the energy operator of the model and $\nu=2$ for all the models.
\end{abstract}

PACS numbers: 03.67.Mn, 05.50.+q, 05.65.+b

\section{INTRODUCTION}

Usually, we are interested in ground state properties of quantum critical chains in the thermodynamic limit. However, obtaining the physical properties in this limit, in general, is not possible. On the other hand, many numerical techniques that give accurate information about the system, work for finite systems. It is therefore of fundamental importance to obtain the physical properties of the infinite system of interest from its formulation in a finite-size geometry. Indeed, various physical quantities of interest are directly related to the finite-size scaling corrections of some physical measure. In this work, we are interested in the scaling corrections of the entanglement entropy of one-dimensional critical systems, which has been intensely debated in recent years.

Consider a quantum chain with $L$ sites, described by a pure state whose density operator is $\rho$. Let us consider that the system is composed by the subsystems $\mathcal{A}$ with $\ell$ sites $(\ell=1, \ldots, L)$ and $\mathcal{B}$ with $L-\ell$ sites. The Rényi entropy is defined as

$$
S_{\alpha}(L, \ell)=\frac{1}{1-\alpha} \ln \operatorname{Tr}\left(\rho_{\mathcal{A}}^{\alpha}\right),
$$

where $\rho_{\mathcal{A}}=\operatorname{Tr}_{\mathcal{B}} \rho$ is the reduced density matrix of the subsystem $\mathcal{A}$. The von Neumann entropy, also known as entanglement entropy, is the particular limit $\alpha \rightarrow 1$.

In the scaling regime $1<<\ell<<L$, it is expected that for the critical one-dimensional systems, under periodic boundary conditions (PBC), the Rényi entropy of the ground state behaves as

$$
S_{\alpha}(L, \ell)=S_{\alpha}^{C F T}(L, \ell)+S_{\alpha}^{U C S}(L, \ell) .
$$

The first term in this equation, is the conformal field theory (CFT) prediction and is given by $\underline{\underline{1}} \underline{\underline{\underline{ }}}$

$$
S_{\alpha}^{C F T}=\frac{c}{6}\left(1+\frac{1}{\alpha}\right) \ln \left[\frac{L}{\pi} \sin \left(\frac{\pi \ell}{L}\right)\right]+d_{\alpha},
$$

where $c$ is the central charge and $d_{\alpha}$ is a non-universal constant.

Laflorencie et al in Ref. 6 were the first to notice, in an investigation of the spin- $1 / 2$ Heisenberg chain under open boundary condition, that unusual corrections to scaling appear in the Rényi entropy $S_{\alpha}(L, \ell)$ with $\alpha=1$. They noted that the standard CFT term [Eq. 3] could not explain a strong oscillation observed in the von Neumann entropy, which seems to have origin its in the oscillating behavior of the spin-spin correlations of the Hamiltonian. These strong oscillations, for the quantum open chains, were observed subsequently by several authors,$\underline{6}-12$ More recently, Calabrese et al. in Ref. 9 (see also Ref. 10) investigated the anisotropic spin- $1 / 2$ Heisenberg chain with PBC and they noted that although those oscillations are not present in the von Neumann entropy $S_{1}$, they are still present for the Rényi index $\alpha>1$. Based on exact results and also in numerical calculations of the spin1/2 XXZ chain with PBC and zero magnetic filed, Calabrese and collaborators conjectured that $S_{\alpha}^{U C S}$, apart from a non-universal constant $g_{\alpha}$, has the following universal behavior ${ }^{-1}-11$ (see also Ref. 6 )

$$
S_{\alpha}^{U C S}=D_{\alpha}-d_{\alpha}=g_{\alpha} \cos (\kappa \ell)\left[\sin \left(\frac{\pi \ell}{L}\right)\right]^{-p_{\alpha}},
$$

where for convenience we introduce the function $D_{\alpha}(L, \ell)$. In (4) $p_{\alpha}$ is a critical exponent and $\kappa$, which has distint values for different models, gives the spatial period $\lambda=\frac{2 \pi}{\kappa}$ of the oscillations $\frac{13}{2}$ For instance, for the 
spin- $s X X Z$ chains at zero magnetic field $\kappa_{X X Z}=\pi$. For these chains, the universal exponent is $p_{\alpha}=\frac{2 K}{\alpha}$, where $K$ is the Luttinger liquid parameter of the underlying CFT $\stackrel{9,14}{=}$ For the Ising model $\kappa_{I s i n g}=2 \kappa_{X X Z}$, and we see no oscillations. The origin of the oscillating factor $[\cos (\kappa \ell)]$ was not yet completely understood, however it has been observed in the systems whose spin correlations show an oscillating behavior.

By now, the observation of the unusual corrections to scaling in entanglement entropy with the predicted exponents [Eq. (44)] were confirmed only for: the spin-s XXZ chains, $\stackrel{9,14}{,}$ the $1 \mathrm{D}$ attractive Hubbard model, and in a dipolar boson quantum chain. ${ }^{15}$ As observed by Cardy and Calabrese in Ref. 16 the origin of the exponent $p_{\alpha}$ in Eq. (41) is the conical singularities produced by the conformal mapping used to describe the reduced density matrix $\rho_{\mathcal{A}}=\operatorname{Tr}_{\mathcal{B}} \rho$ in CFT. This exponent is related to the scaling dimension $X^{\text {con }}$ of an operator of the underlying CFT by $p_{\alpha}=2 X^{c o n} / \alpha \stackrel{16}{~}$. This operator can be relevant $\left(X^{\text {con }}<2\right)$ or not $\left(X^{\text {con }} \geq 2\right)$. Beyond these corrections, we may also have the usual ones coming from the irrelevant operators (dimension $X^{I}>2$ ), whose leading contributions to (4) are of order $\ell^{-2\left(X^{I}-2\right)}$, for any $\alpha \underline{\underline{16}}$

Recently, Calabrese and Essler in Ref. 10, based on an exact calculation for the XX quantum chain, found the appearance of a correction term of order $\ell^{-2}$ for any value of $\alpha$. As discussed in Ref. 16, we should expect $\alpha$-independent correction terms from the irrelevant operators. This would imply that the contributing irrelevant operator has dimension $X^{I}=3$. Although such an irrelevant operator exists in the conformal tower of the XX model, it is quite unlikely that this contribution comes from such an operator. In this model, the operator responsible for the leading finite-size corrections of the eigenenergies has dimension $X^{I}=4, \frac{17}{1}$ which would produce a correction term of order $\ell^{-4}$. A possible explanation is that the conical singularities may also produce an $\alpha$-independent contribution to the corrections to scaling, besides the term of order $\ell^{-2 X^{\text {con }} / \alpha}$. Although not completely clear, such contribution might come from the combination of contributions of the relevant operators,$\frac{18}{,}$ and in the case of the XX chain it ends up with the main contribution of order $\ell^{-\nu}$, with $\nu=2$.

Although the CFT analysis predicts the possible finitesize corrections of the Rényi entropies, several general questions remain to be answered for a better understanding: a) Among the possible relevant operators in the CFT what should be the leading one, with dimension $X^{\text {con }}$, responsible for the corrections coming from the conical singularities giving $\alpha$-dependent contributions of order $\ell^{-2 X^{c o n} / \alpha}$ ? b) What should be the leading irrelevant operator with dimension $X^{I}$, giving the contribution $O\left(\ell^{-2\left(X^{I}-2\right)}\right)$ ? c) Are the $\alpha$-independent corrections $\ell^{-\nu}$, coming from the conical singularities, as happened in the XX case, general ? In this case what is the value of $\nu$ ? d) The oscillating behavior observed in the $\alpha>1$ Rényi entropy is absent in the quantum Ising chain.
Should we obtain the oscillatory behavior of the entropies for other quantum chains with $U(1)$ symmetries, like the XXZ chain?

In order to test the above predictions and answer the above questions, we present in this paper the calculation of the corrections to scaling in the entropies of several critical quantum chains belonging to distinct universality classes of critical behavior. We consider models with $U(1), Z(2)$ and $Z(3)$ symmetries. The models with $\mathrm{U}(1)$ symmetry are the spin-1/2 XXZ quantum chain and the spin-1 Fateev-Zamolodchikov model. $\underline{19}$ The models with $Z(2)$ and $Z(3)$ symmetries we consider are the quantum Blume-Capel and the quantum 3-state Potts model, respectively.

\section{RESULTS}

From the discussions presented in the introduction we expect, in the region where the system size $L$ and subsystem size $\ell$ are large $(L>>\ell>>1)$, the general behavior:

$$
\begin{aligned}
& D_{\alpha}(L, \ell)=d_{\alpha}+g_{\alpha} \cos (\kappa \ell)\left[\sin \left(\frac{\pi \ell}{L}\right)\right]^{-2 X^{c o n} / \alpha} \\
& +a_{\alpha}\left[\sin \left(\frac{\pi \ell}{L}\right)\right]^{-\nu}+b_{\alpha}\left[\sin \left(\frac{\pi \ell}{L}\right)\right]^{-2\left(X^{I}-2\right)}+\cdots(5)
\end{aligned}
$$

The second and third terms are the leading contributions due to the conical singularities, $\frac{16}{=}$ and the last term is the leading contribution due to the standard correction to scaling operator, with dimension $X^{I}$. The operator that produces the second term in (6) has dimension $X^{\text {con }}$, while the relation of the $\alpha$-independent exponent $\nu$ with the dimension of operators is unknown. Models exhibiting oscillations in the $\alpha$-entropies have $g_{\alpha} \neq 0$ and $\kappa \neq 0$ $(\bmod 2 \pi)$. Note that only the exponent of the second leading corrections depend on the value of $\alpha$. We intend to evaluate the power of the dominant term in (5), which we denote by $p_{\alpha}$. In order to estimate the exponent $p_{\alpha}$, we fit our data with the following equation

$$
D_{\alpha}(L, \ell)=d_{\alpha}+f_{\alpha}[\cos (\kappa \ell)]^{\left(1-\delta_{\alpha, 1}\right)}\left[\sin \left(\frac{\pi \ell}{L}\right)\right]^{-p_{\alpha}} .
$$

We report first the results already known from earlier studies for some models. The spin- $1 / 2 \mathrm{XXZ}$ chain shows oscillations for $\alpha>1$. These corrections come from the operator with dimension $X^{\text {con }}=K$, where $K$ is the Luttinger liquid parameter ${ }^{9}$ This is the scaling dimension of the energy operator of the model $\stackrel{20}{2}$ For $\alpha=1$ the oscillations do not appear, i. e., $g_{1}=0$, and the dominant term in (5) is not known for general values of the anisotropy of the model. In the particular case where we have the XX model (free fermion case), the dominant correction for $D_{1}(L, \ell)$ is given by the third term in (6) with $\nu=2 \underline{10}$

The non integrable spin- $s$ XXZ chain $(s=1,3 / 2, \ldots)$ on its critical regions also shows entropy oscillations for 
$\alpha>1$, but the amplitudes $g_{\alpha}$ decreases strongly as we increase the value of the spin $s \stackrel{14}{\underline{14}}$ On these models, the amplitudes of the entropy oscillations are also ruled by the energy operator of the model, with dimension $X_{\epsilon}=X^{c o n}=K$, given by the Luttinger parameter of the underlying CFT.

The results for the Ising model can be obtained from those of the spin- $1 / 2 \mathrm{XX}$ model due to the exact correspondence of their entropies, as shown in Ref. 21. The Ising model does not show oscillations, for any $\alpha$, due to the fact that $\kappa_{I s i n g}=2 \kappa_{X X Z}=2 \pi$. We also have $g_{1}=0$ and $g_{\alpha} \neq 0(\alpha>1)$ with the dominant correction term given by $X^{\text {con }}=1$, which is also the dimension of the energy operator of the model. In the case $\alpha=1$ the dominant correction comes from the third term of (5) with $\nu=2$.

Below, we present new numerical results for three models: (i) the Blume-Capel Model (BCM), (ii) three-state Potts Model (3SPM), and (iii) the Fateev-Zamolodchikov quantum chain (FZQC). We investigated these models with the density-matrix renormalization group (DMRG) technique ${ }^{22}$ under PBC. We have done 4-8 sweeps. For the BCM and the 3SPM we used typically $m=400$ states per block. This number of states kept in the truncation process is enough to give very precise results, the discarded weight being typically about $10^{-12}$. However, for the FZQC we used a much larger number of states in order to obtain precise results (up to $m=3000$ ). In this case, the discarded weight was typically $10^{-8}$ in the final sweep.

\section{A. The spin-1 Blume-Capel model}

We begin by introducing the spin-1 BCM quantum chain. This model is obtained by the time-continuum limit of the well known BCM in two dimensions $\stackrel{23}{\text { It de- }}$ scribes the dynamics of spin-1 localized particles, with Hamiltonian given by

$$
H_{B C}=-\sum_{j}\left(s_{j}^{z} s_{j+1}^{z}-\delta\left(s_{j}^{z}\right)^{2}-\gamma s_{j}^{x}\right),
$$

where $s^{x}$ and $s^{z}$ are the spin-1 $S U(2)$ operators. The phase diagram, in the $\delta-\gamma$ plane, is known from earlier numerical finite-size scaling studies based in the crossing of the mass gap energies (see Fig. 1 of Ref. 23). For values $\gamma>\gamma_{\text {tr }}$ the Hamiltonian has a quantum critical line $\delta_{c}(\gamma)$ governed by a CFT in the same universality class of the quantum Ising chain, i. e., central charge $c=1 / 2$. At $\gamma_{\mathrm{tr}}$ the model has a quantum tricritical point at $\delta_{\text {tr }}$ in the universality class of the tricritical Ising model, having central charge $c=7 / 10$. For $\gamma<\gamma_{\text {tr }}$ there is a line $\delta=\delta_{\text {gap }}(\gamma)$ of first-order phase transitions. Recently, highly accurate estimates of some points of the critical line were obtained by a new method based on the entanglement entropy $\stackrel{24}{=}$ In particular, the tricritical point was located at $\gamma_{\mathrm{tr}}=0.41563$ and $\delta_{\mathrm{tr}}=0.91024$.

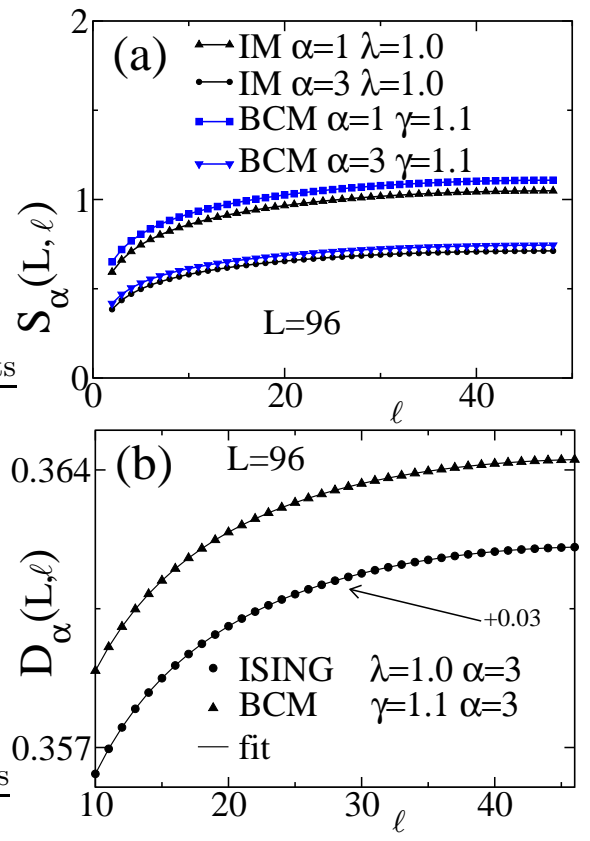

Figure 1: (Color online). Results of the Rényi entropy $S_{\alpha}(L, \ell)$ and of the function $D_{\alpha}(L, \ell)$ for the Ising and BlumeCapel models with PBC and size $L=96$. (a) $S_{\alpha}(L, \ell)$ vs $\ell$ for two values of $\alpha$, and some values of coupling (see legend). The symbols are the numerical data and the solid lines connect the fitted points using Eq. (3). (b) $D_{\alpha}(L, \ell)$ vs $\ell$ for $\alpha=3$. We added 0.03 in the values of the Ising entropy in order to see better both data in the same figure.

Since the model has a critical line in the universality class of the Ising model (IM) it is interesting, for the sake of comparison and as benchmark tests, to consider also the integrable IM. The Hamiltonian of the IM is given by

$$
H_{\text {Ising }}=-\sum_{j}\left(\sigma_{j}^{x} \sigma_{j+1}^{x}+\lambda \sigma_{j}^{z}\right)
$$

where $\sigma^{x}, \sigma^{z}$ are spin- $1 / 2$ Pauli matrices. This model has a critical point $\lambda_{c}=1$ that can be obtained from its exact solution, or even more simply from its self dual property 25

In Fig. 1(a), we show the Rényi entropy $S_{\alpha}(L, \ell)$, as a function of $\ell$, for the IM and the BCM with PBC and lattice size $L=96$. In the IM case, we select the exact critical point $\lambda=1$. Since in the BCM we do not know the exact values of the critical points, we used the best estimate of the critical points available from previous work ${ }^{24} \mathrm{In}$ particular, for $\gamma=1.1$ we used $\delta_{c}=0.31357$, where we expect an error in the last digit (see Ref. 24). In both cases, the fits to Eq. (3) give a central charge value very close to the exact one. We found that $\left|c^{f i t}-c^{\text {exact }}\right| \lesssim 10^{-3}$ for all critical couplings considered (some of these estimates are presented in Table III). We emphasize that the discrepancies between the exact values and the numerical data are due to the finite-size of the systems considered. The numerical errors (inferred from the dependence of the en- 
tropy with $m$ ) are smaller than the size of the symbols in the figures. For the models with discrete (continuous) symmetry the errors are smaller than $10^{-5}\left(10^{-3}\right)$.

The results presented in Fig. 1(a) are nice illustrations of the universal behavior of the Rényi entropies at the critical points. Although the entropies are calculated from ground states defined in Hilbert spaces of quite distinct dimensions $\left(2^{L}\right.$ and $\left.3^{L}\right)$ the entropies show the same behavior. The small deviation shown in this figure is due to the non-universal constant $d_{\alpha}$ in (3). Notice also that both models, in the Ising Universality class, show no oscillations in the entropies (even in the OBC case, not shown), i. e., $\kappa=0$ in (5).

The corrections to scaling contributions are calculated from the behavior of $D_{\alpha}(L, \ell)$ given in (5). As illustrative examples, let us consider, as in Fig. 1(a), the IM at the critical coupling $\lambda_{c}=1$ and the BCM at the couplings $\gamma=1.1$ and $\delta_{c}=0.31357$. In Fig. 1(b), we present $D_{\alpha}(L, \ell)$ for the critical couplings of the quantum chains with PBC, $\alpha=3$, and lattice size $L=96$ (similar results are found for $L=64$ ). Since at these couplings, both models have central charge $c=1 / 2$ we set this value in Eq. (3). Note in these two figures that the changes in $D_{\alpha}(L, \ell)$, as function of $\ell$, are quite small, which indicate that the non-universal constants $g_{\alpha}, a_{\alpha}$ and $b_{\alpha}$ that appear in (5) are very small. This fact makes the task of determining the exponent $p_{\alpha}$ in (6) a challenge. Since we are interested in the asymptotic behavior of $D_{\alpha}(L, \ell)$, we have discarded the first 10 values of $\ell$. Comparison of the entropies with different values of $m$ indicate that the errors in the DMRG evaluations of the entropies are around $\sim 10^{-6}-10^{-7}$. Due to this fact, we also discarded the sites $\ell$ at which $\left|D_{\alpha}(L, \ell)-D_{\alpha}(L, \ell+1)\right|<10^{-5}$.

We get $p_{\alpha}$ by fitting our data to Eq. (6). The solid lines, in Fig. I(b), connect the fitted points. In order to check if we are able to extract good estimates of $p_{\alpha}$ with the above mentioned procedure, we compare the values of $p_{\alpha}$, acquired with the fit procedure with the exact ones $\left(p_{\alpha}=\frac{2}{\alpha}\right)$ for the Ising model. The finite-size estimates for the exponents $p_{2}, p_{3}$ and $p_{4}$ are depicted in Table I. As we can observe in this table, the finitesize estimates differ very little (less than $5 \times 10^{-2}$ ) from the exacts values. We then believe that this approach will also provide good estimates of the exponents $p_{\alpha}$ for other models like the BCM.

Before presenting our results for the BCM, it is interesting to mention that, for the IM, we got only 8 points of $D_{\alpha=1}^{I \operatorname{sing}}(L, \ell)$ satisfying $\left|D_{1}(L, \ell)-D_{1}(L, \ell+1)\right|<10^{-5}$ for $\ell>2$. This in fact is expected, since for $\alpha=1$ in the IM, the dominant correction to scaling is conjectured as $D_{1}(L, \ell)=d_{1}+\frac{1}{60} \frac{\pi^{2}}{L \sin (\pi \ell / L)]^{2}}+\frac{\pi^{2}}{18 L^{2}}, \frac{26}{,}$ which has a larger exponent than the ones of $\alpha>1$. It is amazing that a fit of those 8 points to Eq. (6) give the following values: $d_{1}=0.478, f_{1} \times(L / \pi)^{2}=0.018$ and $p_{1}=2.05$. These values are very close to the exact ones, i. e.: $d_{1}=0.4785$, $f_{1} \times(L / \pi)^{2}=1 / 60=0.0166$ and $p_{1}=2.26 .27$

It is interesting to stress that the correction term of order $\ell^{-2}$ in the case of the IM does not come from the

\begin{tabular}{ccc}
$p_{2}$ & $p_{3}$ & $p_{4}$ \\
\hline \hline 0.978 & 0.646 & 0.473 \\
$(1.000)$ & $(0.6666)$ & $(0.5000)$ \\
\hline \hline
\end{tabular}

Table I: The finite-size estimates of the exponents $p_{\alpha}$ obtained from the fit of Eq. (6) for the Ising model with PBC and $L=96$. The results in parentheses are the exact values.

second term in (5) with $\kappa_{\text {Ising }}=2 \pi$, but from the third term with $\nu=2$. This is due to the exact relations among the entropies of the IM and the XX chain, and the fact that there is no oscillations in the last model for $\alpha=1$, i. e. $g_{1}=0$.

Let us now focus on the BCM. We show in the last column of Table $\amalg$ the finite-size estimates of the central charge $c$ for three values of $\gamma$ along the critical line. We got these estimates by a simple fit of our data to Eq. (3) for $\alpha=1$. As we note, theses values of $\gamma$ satisfy $\gamma>\gamma_{\text {tr }}=0.41563$, and the system belongs to the same universality class of critical behavior as the Ising model with $c=1 / 2$. Following the same procedure done in the IM, we fit our data to Eq. (6) with the appropriated values of $c$ [see Fig. 1(b)], in order to extract the exponents $p_{\alpha}$. Some finite-size estimates of $p_{\alpha}$ are depicted in Table II As observed in this table, for $\gamma>\gamma_{\mathrm{tr}}$, our results are consistent with the exponent $p_{\alpha}=2 / \alpha$, which is the same of the IM. In the case $\alpha=1$, where the evaluations are more difficult we got, in the BCM, around 10 points of $D_{\alpha}(L, \ell)$ satisfying $\left|D_{1}(L, \ell)-D_{1}(L, \ell+1)\right|<10^{-5}$ for $\ell>5$, and the fit to Eq. (6) indicate that $p_{1}=2$. Since the energy operator $X_{\epsilon}=1$, this term could be produced either by the first term in (5) with $g_{1} \neq 0$ but $\kappa=0$, or by the second term in (5) with $\nu=2$. However, the exact result derived for the IM, that we expect to be the same for any model on its universality class, indicate that $g_{1}=0$ and the dominant term in (5) is the one with exponent $\nu=2$. Our estimates for this exponent $p_{1}=\nu$ are shown in Table $\amalg$

At the tricritical point $\left(\gamma_{\text {tric }}, \delta_{\text {tric }}\right)$, we were not able to extract the exponents $p_{\alpha}$ due to the fact that the $\alpha$ entropies are very sensitive to the coupling constants of the model. We observed that a very small error $(\epsilon \sim$ $\left.10^{-4}\right)$ in the critical coupling $\delta_{c}$ affects very little the value of the entropy along the critical line. Typically, the entropy changes are of the same magnitude of $\epsilon$. On the other hand, at the tricritical point, a small change in the critical couplings produces a much larger effect. Due to this fact, since the precision of the tricritical couplings are $\sim\left(10^{-5}\right)$, which is of the same order of magnitude as $D_{\alpha}$, we are not able to obtain a reasonable estimate of $p_{\alpha}$ at the tricritical point. 


\begin{tabular}{|c|c|c|c|c|c|}
\hline$\gamma$ & $p_{2}$ & $p_{3}$ & $p_{4}$ & $\nu$ & $c$ \\
\hline 1.2 & 1.066 & 0.697 & 0.517 & 2.071 & 0.5000 \\
\hline 1.1 & 1.041 & 0.682 & 0.504 & 2.062 & 0.5001 \\
\hline 0.7 & 1.154 & 0.776 & 0.589 & 1.955 & 0.5015 \\
\hline & (1) & $(2 / 3)$ & $(1 / 2)$ & (2) & $(1 / 2)$ \\
\hline
\end{tabular}

Table II: The finite-size estimates of the exponents $p_{\alpha}$ acquired by the fit of Eq. (6) for the BCM with PBC, $L=96$, and some values of $\gamma$. In the last two columns we also present the finite-size estimates of the conformal anomaly $c$, and the exponent $p_{1}=\nu$ (see text). The values in parentheses are the expected ones.

\section{B. The three state Potts Model}

The quantum $3 \mathrm{SPM}$ is a quantum chain obtained by the $\tau$-continuum limit of the two dimensional classical $3 \mathrm{SPM}, \underline{28}$ The quantum Hamiltonian of the model can be written as

$$
H_{\text {Potts }}=-\sum_{i}\left(R_{i} R_{i+1}^{\dagger}+\text { h.c. }\right)+\lambda O_{i}
$$

where $R$ and $O$ are $3 \times 3$ matrices given by

$$
R=\left(\begin{array}{lll}
0 & 1 & 0 \\
0 & 0 & 1 \\
1 & 0 & 0
\end{array}\right) \quad O=\left(\begin{array}{ccc}
2 & 0 & 0 \\
0 & -1 & 0 \\
0 & 0 & -1
\end{array}\right)
$$

As the IM, due to its self duality the 3SPM also has the critical point at $\lambda_{c}=1$. However, the critical fluctuations of the 3SPM are described by a CFT with $c=4 / 5$. For PBC, as a consequence of the modular invariance of the related two dimensional model defined on a cylinder, the associated CFT is described in terms of 10 primary operators $\frac{29}{2}$ Among these operators, we focus on the most relevant operators, which may lead to the unusual corrections to scaling in the entropy.

The first nonzero dimension $\left(X_{m}=2 / 15\right)$ is associated to the order parameter and the second one $\left(X_{\epsilon}=4 / 5\right)$ is associated to the energy operator. The lowest irrelevant operator responsible for the finite-size corrections of the eigenenergies of the Hamiltonian has dimension $X^{I}=$ $14 / 5, \underline{17,29}$

Note that in the IM and the BCM, the leading corrections to scaling in the $\alpha$-entropies with $\alpha>1$ are related to the dimension of the energy operator $X_{\epsilon}$. For this reason, we expected in the 3SPM that $p_{\alpha}=\frac{2 X_{\epsilon}}{\alpha}=\frac{8}{5 \alpha}$ for $\alpha>1$. Below we use the same procedure used in the IM and the BCM to get the exponent $p_{\alpha}$.

In Fig. 2(a), we present the Rényi entropy as a function of $\ell$ for the 3SPM at the critical coupling $\lambda_{c}=1$ for a system with lattice size $L=96$. As observed in this figure, we also do not see the parity oscillations, like the
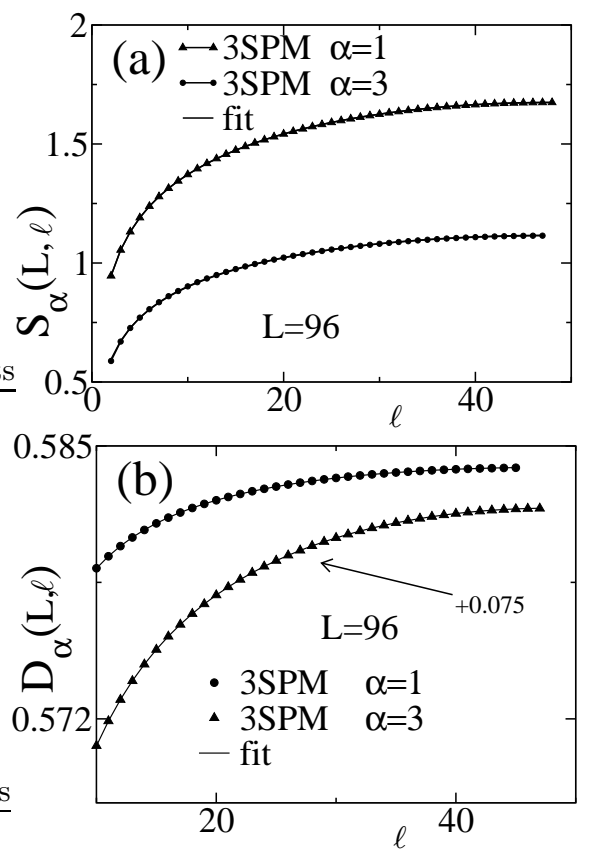

Figure 2: (Color online). Rényi entropy $S_{\alpha}(L, \ell)$ and the difference $D_{\alpha}(L, \ell)$ for the $3 \mathrm{SPM}$ with lattice size $L=96$. The symbols are the numerical data and the solid lines connect the fitted points (see text). (a) $S_{\alpha}(L, \ell)$ vs $\ell$ for $\lambda=1$, and some values of $\alpha$ (see legend). (b) $D_{\alpha}(L, \ell)$ vs $\ell$ for $\lambda=1, \alpha=2$ and $\alpha=3$.

models considered in the last section (which also have a discrete symmetry). We are able, in this case, to obtain a nice fit of our data by considering only the standard term of the CFT [Eq. (3)]. The solid lines in these figures connect the fitted points. In this case, we get $c=0.799$ (see Table III) very close to the expected value $c=4 / 5$.

Finally, we depicted in Table III the exponents $p_{\alpha}$ obtained through a fit of our data to Eq. (6). As we can see in this table our numerical results indicate that, for $\alpha>1, p_{\alpha}=\frac{8}{5 \alpha}$ confirming our prediction that like in the IM and $\mathrm{BCM}$ the energy operator gives the most important contribution in the conical singularities. In the case of the von Neumann entropy, i. e. $\alpha=1$, our numerical results indicate that the leading corrections are given by the third term in (6) with the parameter $\nu=2$. As happened in the Ising universality class the operator that gives the leading contribution for $\alpha>1$, that we believe to be the energy operator, does not contribute for $\alpha=1$, i. e., $g_{1}=0$. Moreover, since the leading irrelevant operator is $X^{I}=14 / 15$, we would expect a contribution of order $[\sin (\pi \ell / L)]^{-8 / 15}$ in (5), which is also not present, i. e., $b_{1}=0$. This implies that both the energy and the leading irrelevant operator ruling finite-size corrections do not contribute, at least at leading order, to the usual von Neumann entropy. The result $g_{1}=0$ found for all models discussed above indicate that, probably, this is a general behavior for quantum critical chains. 


\begin{tabular}{cccc}
$p_{2}$ & $p_{3}$ & $p_{4}$ & $c$ \\
\hline \hline 0.829 & 0.523 & 0.371 & 0.799 \\
$(0.800)$ & $(0.5333)$ & $(0.400)$ & $(0.800)$ \\
\hline \hline
\end{tabular}

Table III: The finite-size estimates of the exponents $p_{\alpha}$ acquired by fit of Eq. (6) for the three state Potts model for $L=96$. In the last column we also present the finite-size estimates of the conformal anomaly $c$ (see text). The results in parentheses are the expected values.

\section{The spin-1 Fateev-Zamolodchikov quantum chain}

The spin-1 Fateev-Zamolodchikov quantum chain (FZQC) is an exact integrable quantum chain whose Hamiltonian is given by 19

$$
\begin{aligned}
H_{F Z} & =\epsilon \sum_{j}\left\{\mathbf{s}_{j} \cdot \mathbf{s}_{j+1}-\left(\mathbf{s}_{j} \cdot \mathbf{s}_{j+1}\right)^{2}\right. \\
& +4 \sin ^{2}(\delta / 2)\left(T_{j}^{\perp} T_{j}^{z}+\text { h.c. }\right) \\
& \left.-2 \sin ^{2}(\delta)\left[T_{j}^{z}-\left(T_{j}^{z}\right)^{2}+2\left(s_{j}^{z}\right)^{2}\right]\right\},
\end{aligned}
$$

where $\epsilon= \pm 1, T_{j}^{z}=s_{j}^{z} s_{j+1}^{z}, T_{j}^{\perp}=s_{j}^{x} s_{j+1}^{x}+s_{j}^{y} s_{j+1}^{y}$, and $s_{j}^{x}, s_{j}^{y}, s_{j}^{z}$ are the spin-1 $S U(2)$ operators.

This Hamiltonian has a $U(1)$ symmetry, having the $z$ magnetization as a good quantum number. The antiferromagnetic $(\epsilon=1)$ and ferromagnetic $(\epsilon=-1)$ models show a critical phase with continuously varying exponents for $0 \leq \delta \leq \pi / 2$. The antiferromagnetic and ferromagnetic models are governed by a CFT with central charge $c=3 / 2$ and $c=1$, respectively $\underline{30}$ The CFT in the $c=3 / 2$ case is described in terms of composite fields formed by Gaussian and Ising operators $30-33$ The energy operator, which we believe is responsible for the leading contributions due to the conical singularities, has dimension $X_{\epsilon}=\frac{\pi}{4(\pi-2 \delta)}+\frac{1}{8}$ and the leading irrelevant operator responsible for the finite-size corrections of the eigenenergies has dimension $X^{I}=\frac{\pi}{\pi-2 \delta}+1, \underline{31,32}$

In the case $c=1$, we have a Gaussian CFT or a standard Luttinger liquid, whose Luttinger parameter changes continuously along the critical line. In this case, the dimension of the energy operator is $X_{\epsilon}=\frac{\pi}{2 \delta}$ and the leading irrelevant operator responsible for the corrections to scaling has dimension $X^{I}=\frac{2 \pi}{\delta}, 34$

In Figs. 3(a)-(b), we present the Rényi entropy $S_{\alpha}(L, \ell)$ for spin-1 FZQC with, PBC, $\delta=0.5$, and size $L=72$. Notice that for $\epsilon=1$, differently from the Ising and the Blume-Capel models, the $\alpha$-Rényi entropies oscillate for $\alpha>1$, which is a signature of the oscillating spin-spin correlations of the quantum chain. The absence of oscillations for $\alpha=1$ also suggests that like the other models we have studied in previous subsections $g_{1}=0$ in (57). Let us focus, first, on the case $\alpha=1$. In this case, we get very nice fit of our numerical data (black triangles) to
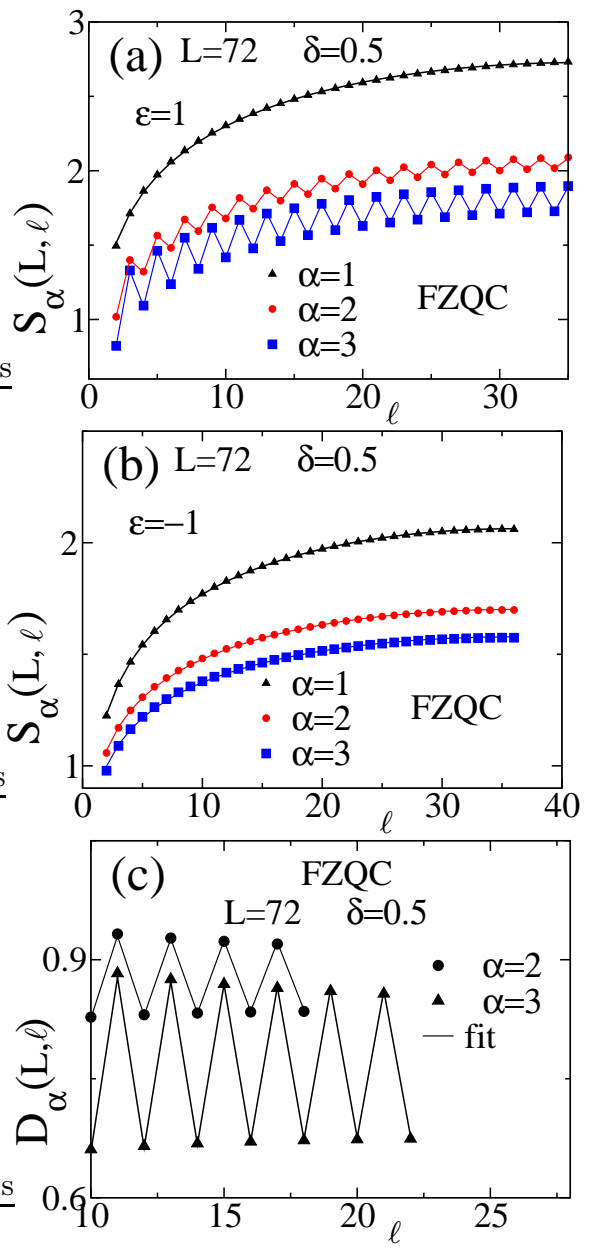

Figure 3: (Color online). Results of the Rényi entropy $S_{\alpha}(L, \ell)$ and the difference $D_{\alpha}(L, \ell)$ for the spin-1 FateevZamolodchikov quantum chain with $\mathrm{PBC}$ and size $L=72$. The symbols are the numerical data and the solid lines connect the fitted points (see text). (a) $S_{\alpha}(L, \ell)$ vs $\ell$ for $\epsilon=1$, $\delta=0.5$, and some values of $\alpha$ (see legend). (b) Same as (a) but for $\epsilon=-1$. (c) $D_{\alpha}(L, \ell)$ vs $\ell$ for $\epsilon=1, \alpha=2$ and $\alpha=3$ at $\delta=0.5$.

the standard conformal field term $S_{\alpha}^{C F T}$ [Eq. (3)]. The fit gives us $c=1.491(c=1.02)$ for $\epsilon=1(\epsilon=-1)$, which is very close to the exact value $c_{\text {exact }}=3 / 2\left(c_{\text {exact }}=1\right)$. Similar estimates of $c$ were also obtained by considering $\alpha=2$ or $\alpha=3$. Note that the central charge $c$ of the FZQC is larger than the ones of Ising and Blume-Capel models. This means that the subsystem $\mathcal{A}$ is more entangled with the subsystem $\mathcal{B}$ in the FZQC, as compared with the critical Ising or Blume-Capel models. Due to this fact, we have to keep a much larger number of states $m$ (typically around $m \sim 2000-3000$ ) for the FZQC, in order to get results with some similar accuracy.

Now let us consider the antiferromagnetic model $\epsilon=1$ and $\alpha>1$. In this case, it is not possible to fit the numerical data if we consider only the standard CFT term $S_{\alpha}^{C F T}$ [Eq. (33)]. Relative nice fits are obtained only if we consider besides the CFT term, the oscillatory 


\begin{tabular}{c|ccc}
$\delta$ & $p_{2}$ & $p_{3}$ & $p_{4}$ \\
\hline \hline 0.25 & 0.413 & 0.286 & 0.215 \\
& $(0.4223)$ & $(0.2815)$ & $(0.2112)$ \\
& & & \\
0.5 & 0.432 & 0.304 & 0.231 \\
& $(0.4917)$ & $(0.3278)$ & $(0.2459)$ \\
1.0 & & & \\
& 0.778 & 0.541 & 0.402 \\
& $(0.8129)$ & $(0.5419)$ & $(0.4064)$ \\
\hline \hline & \multicolumn{3}{l}{}
\end{tabular}

Table IV: The finite-size estimates of the exponents $p_{\alpha}$ acquired by fit of Eq. (6) for the spin-1 Fateev-Zamolodchikov quantum chain with $\mathrm{PBC}, L=72$ and some values of $\delta$. The values in parentheses are the expected ones (see text).

correction term [Eq. (4)] with $\kappa=\pi / 2$. The solid lines in Fig. 3(a) are the fits to Eq. (2). For instance, for $\alpha=2(\alpha=3)$ we get $c=1.48(c=1.45)$ and $p_{2}=0.54$ $\left(p_{3}=0.36\right)$.

Since we are confident that $c=3 / 2$ for the spin- 1 FZQC, we set $c=3 / 2$ in Eq. (3), as well $\kappa=\pi / 2$ in the function $D_{\alpha}(L, \ell)$ [Eq. (5)], in order to evaluate the exponent $p_{\alpha}$ in the same way we did in the Ising, Blume-Capel and three state Potts models. In Fig. 3(b), we show $D_{\alpha}(L, \ell)$, as function of $\ell$, for the spin-1 FZQC with $\mathrm{PBC}, \epsilon=1, \delta=0.5, L=72$ and three values of $\alpha$. Even keeping $m=3000$ states in the DMRG, it is very hard to get high precision in the values of the entropy for the spin-1 FZQC, as we already mention before. Although the truncation errors are around $\sim 10^{-8}$, comparison of the entropy results with different values of $m$, indicate that the errors in the entropy are around $\sim 10^{-3}$. For this reason, we discarded the points that $\left|D_{\alpha}(L, \ell)-D_{\alpha}(L, \ell+2)\right|<10^{-3}$, as well as the first 10 sites. The solid lines in this figure connect the fitted points. The finite-size estimates of $p_{\alpha}$ in (6), obtained by this fitting procedure, are depicted in Table IV The results presented in this table indicate that $p_{\alpha}=2 X_{\epsilon} / \alpha$, where $X_{\epsilon}=\frac{\pi}{4(\pi-2 \delta)}+\frac{1}{8}$ is the dimension of the energy operator of the model. This means that, like the other quantum chains, the energy operator gives the most important contribution in the conical singularities for $\alpha>1$. In the case where $\alpha=1$, like the other models, this contribution seems to be absent, i. e., $g_{1}=0$ [see Fig. 33(a)]. In this case, we were not able to estimate numerically the power of the leading correction, since we only have three points satisfying $\left|D_{1}(L, \ell)-D_{1}(L, \ell+2)\right|<10^{-3}$.

In the ferromagnetic case $(\epsilon=-1)$ although we expect oscillations to be present in the $\alpha$-entropies with $\alpha>1$, we could not see them numerically [see Fig. 3(b)]. This is consistent with the conjecture that the oscillations are ruled by the energy operator. In this case the amplitude of the oscillations will decay like $\ell^{-p_{\alpha}}$, $p_{\alpha}=\frac{2 X_{\epsilon}}{\alpha}=\frac{\pi}{\delta \alpha}$. The region where we would better see the oscillations would be for $\delta \approx \frac{\pi}{2}$, where $p_{\alpha}$ has the smaller values. However the sound velocity, which is given by ${ }^{34} v_{s}=\pi \sin (2 \delta) /(2 \pi-2 \delta)$ is close to zero in this region. This makes the convergence quite slow in the DMRG, due to the small size of the energy mass gaps. To avoid this problem and get enough precision, we considered values of $\delta$ in other regions, like $\delta=1 / 2$ for example [see Fig. 3(b)]. However, in this case, the expected value of $p_{\alpha}=2 \pi / \alpha$ is for $\alpha=3, p_{\alpha} \approx 2.1$. This gives a strong decay, large enough to forbid, within the numerical precision we have, the observation of the oscillations for $\ell>10$.

\section{CONCLUSIONS}

Most of the critical quantum chains are conformal invariant. This symmetry implies that the mass gap amplitudes of these critical chains, in a finite lattice, are related to the conformal anomaly and critical exponents that label the particular universality class of critical behavior. ${ }^{35}$ Due to these relations, the most frequently used method to extract the conformal anomaly and critical exponents comes from the finite-size scaling of the eigenenergies of the critical quantum chains.

On the other hand, the conformal anomaly can also be calculated from the Rényi entanglement entropies of the ground state of finite quantum chains, $1,2,5,36$ a property related to the eigenfunctions instead of the eigenenergies. These results raised the question about the possibility to extract all the critical exponents from a complete finitesize study of the Rényi entropies of the critical quantum chains. This question has also practical implications, since in DMRG calculations the entanglement entropies are much simpler to calculate, as compared with the mass gap energies. Unfortunately the leading terms of the entanglement Rényi entropies of the low-lying excited states are the same as that of the ground state $\stackrel{37,38}{=}$ The critical exponents only appear in the finite-size corrections of these entropies $\stackrel{9.16}{=}$ In the finite-size study of the energy gaps the exponents are given by the leading terms and we know precisely the correspondence between the mass gap amplitudes and the dimensions of the underlying CFT. In the finite-size study of the entanglement entropy, since the exponents are given not by the leading term, but by the finite-size corrections, we do not have such exact correspondence. It is known from conformal invariance $\frac{16}{6}$ that these corrections are ruled either by relevant operators, due to the conical singularities in the conformal mapping, or by irrelevant operators. However, it is not known what are those operators, or equivalently what should be the critical exponent that appears in the leading finite-size corrections of the entanglement entropies in general models. The results presented in this paper, together with known results induce us to announce some conjectures about the operators governing the finite-size corrections of the Rényi entropies of critical quantum chains with PBC. We are going to state them separately 


\begin{tabular}{ccccc}
$\Delta$ & $M=0$ & $M=1 / 6$ & $M=1 / 4$ & $M=3 / 10$ \\
\hline 0.5 & 1.9 & 2.0 & 2.0 & 2.1 \\
$\sqrt{2} / 2$ & - & 2.0 & 2.0 & 2.1 \\
0.9980 & - & 1.98 & 2.0 & 2.1 \\
2.0 & - & 1.97 & 2.0 & - \\
\hline \hline
\end{tabular}

Table V: The exponent $\nu$ of the finite-size corrections of the von Neumann entropy for the spin-1/2 XXZ chain with PBC, and some values of $\Delta$ and magnetization per site $M$. We extracted the exponent, as we did for the other models, considering systems with size $L=96$. For value of anisotropy close to the isotropic antiferromagnetic point $(\Delta=1)$ and $M=0$, we were not able to obtain the exponent, mainly due to the large finite-size corrections.

(a) Entropy oscillations in the $\alpha$-Rényi entropies of critical quantum chains only appear for $\alpha>1$ and for models having at least one $U(1)$ symmetry, like the spin- $s$ Heisenberg, $t-J$ and Hubbard models. Models possessing only discrete symmetries like the Ising, Blume-Capel and Potts models show no oscillations for any value of $\alpha$.

(b) For any critical quantum chain the amplitude of the leading correction of the $\alpha$-Rényi entropy with $\alpha>1$ has a universal power decay $p_{\alpha}=2 X_{\epsilon} / \alpha$, where $X_{\epsilon}$ is the dimension of the energy operator of the model. We stress that these results are expected only for the entanglement entropy of a single interval. In the case of two disjoint intervals, the results of Ref. 39 indicate that instead of the energy operator the leading contributions comes from nonlocal spinor operators.

The amplitudes of the leading corrections for $\alpha=1$, have a quite distinct behavior from the $\alpha>1$ cases. For all models we considered these amplitudes have a power law decay $\ell^{-\nu}$, where $\nu=p_{1}=2$. Since these corrections for the $\alpha=1$ Rényi entropy (von Neumann entropy) are not known in the case of the spin- $1 / 2 \mathrm{XXZ}$ chain [anisotropy $\Delta$ ], we present in Table $\mathbf{V}$ those corrections for some values of anisotropy and magnetization per site $M$. We clearly see, that also for the XXZ quantum chain the leading finite-size correction always decay as $\ell^{-2}$. In the case of the XX quantum chain, where $\nu=2$ is an

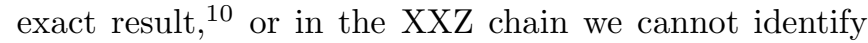
the operator responsible for such finite-size correction. All these results indicate the following conjecture.

(c) The leading finite-size corrections of the $\alpha=1$ Rényi entropy, or the von Neumann entropy, of any quantum chain decays as $\ell^{-2}$. This is the main contribution coming from the conical singularities in the conformal mapping, but we do not know what operators produce such universal correction.

We expect that the above conjectures can be confirmed for other models and we hope they can be understood by using the general properties of the underlying CFT ruling the long-distance physics of quantum chains.

\section{Acknowledgments}

We are in debt to P. Calabrese, F. Essler and R. G. Pereira for discussions and a careful reading of the manuscript. We also thank M. J. Martins and V. Rittenberg for useful discussions. This research was supported by the Brazilian agencies FAPEMIG, FAPESP, and CNPq.
1 C. Holzhey, F. Larsen, and F. Wilczek, Nucl. Phys. B424, 443 (1994)

${ }^{2}$ P. Calabrese and J. Cardy, J. Stat. Mech., P06002(2004)

${ }^{3}$ P. Calabrese and J. Cardy, J. Phys. A: Math. Theor. 42, 504005 (2009)

4 I. Affleck and A. W. W. Ludwig, Phys. Rev. Lett. 67, 161 (1991)

5 V. E. Korepin, Phys. Rev. Lett. 92, 096402 (2004)

6 N. Laflorencie, E. S. Sørensen, M. S. Chang, and I. Affleck, Phys. Rev. Lett. 96, 100603 (2006)

7 M. Fuehringer, S. Rachel, R. Thomale, M. Greiter, and P. Schmitteckert, Ann. Phys. (Berlin) 17, 922 (2008); A. M. Läuchli and C. Kollath, J. Stat. Mech., P05018(2008); A. B. Kallin, I. González, M. B. Hastings, and R. G. Melko, Phys. Rev. Lett. 103, 117203 (2009); Ö. Legeza, J. Sólyom, L. Tincani, and R. M. Noack, ibid. 99, 087203 (2007); G. Roux, S. Capponi, P. Lecheminant, and P. Azaria, Eur. Phys. J. B 68, 293 (2009); I. Affleck, N. Laflorencie, and E. S. Sørensen, J. Phys. A: Math. Theor. 42, 504009 (2009); J. I. Cirac and G. Sierra, Phys. Rev. B 81, 104431 (2010)

8 J. C. Xavier, Phys. Rev. B 81, 224404 (2010)

9 P. Calabrese, M. Campostrini, F. Essler, and B. Nienhuis, Phys. Rev. Lett. 104, 095701 (2010)
10 P. Calabrese and F. H. L. Essler, J. Stat. Mech., P08029(2010)

11 M. Fagotti and P. Calabrese, J. Stat. Mech., P01017(2011)

12 M. Fagotti, P. Calabrese, and J. E. Moore, Phys. Rev. B 83, 045110 (2011)

13 In the case of open boundary condition, an extra phase (model dependent) appears in the cosine oscillating term.

14 J. C. Xavier and F. C. Alcaraz, Phys. Rev. B 83, 214425 (2011)

15 M. Dalmonte, E. Ercolessi, and L. Taddia, Phys. Rev. B 84, $085110(2011)$

16 J. Cardy and P. Calabrese, J. Stat. Mech., P04023(2010)

17 F. C Alcaraz, M. N. Barber, and M. T. Batchelor, Ann. Phys. 182, 280 (1988)

18 F. Essler, private communication.

19 A. B. Zamolodchikov and V. A. Fateev, Solv. J. Nucl. Phys. 32, 2 (1980)

20 There exists in the underlying CFT describing the XXZ quantum chain two primary operators with the same dimension $X^{\text {con }}=K$. The energy operator actually is a combination of these two operators.

21 F. Iglói and R. Juhász, Europhys. Lett. 81, 57003 (2008)

22 S. R. White, Phys. Rev. Lett. 69, 2863 (1992) 
${ }^{23}$ F. C. Alcaraz, J. R. Drugowich de Felício, R. Köberle, and J. F. Stilck, Phys. Rev. B 32, 7469 (1985)

24 J. C. Xavier and F. C. Alcaraz, Phys. Rev. B 84, 094410 (2011)

25 J. Kogut, Reviews of Modern Physics 51, 659 (1979)

26 P. Calabrese, M. Mintchev and E. Vicari, J. Stat. Mech., P09028(2011).

27 F. Iglói and Y. -C. Lin, J. Stat. Mech., P06004(2008)

${ }^{28}$ F. Y. Wu, Rev. Mod. Phys. 54, 235 (1982)

29 J. Cardy, Nucl. Phys. B270, 186 (1986)

30 P. Di Francesco, H. Saleur, and J.-B. Zuber, Nucl. Phys. B 300, 393 (1988)

${ }^{31}$ F. C. Alcaraz and M. J. Martins, Phys. Rev. Lett. 61, 1529 (1988)

32 F. C. Alcaraz and M. J. Martins, J. Phys. A: Math. Gen. 22, 1829 (1989)
33 D. Baranowski and V. Rittenberg, J. Phys. A: Math. Gen. 23, 1029 (1990)

34 F. C. Alcaraz and M. J. Martins, Phys. Rev. Lett. 63, 708 (1989)

35 J. Cardy, Phase transitions and critical phenomena, Vol. 11 (Academic Press, London, 1987)

${ }^{36}$ G. Vidal, J. I. Latorre, E. Rico, and A. Kitaev, Phys. Rev. Lett. 90, 227902 (2003)

37 F. C. Alcaraz, M. I. Berganza, and G. Sierra, Phys. Rev. Lett. 106, 201601 (2011)

38 M. I. Berganza, F. C. Alcaraz, and G. Sierra, arXiv:1109.5673 (2011)

39 V. Alba, L. Tagliacozzo, P. Calabrese, Phys. Rev. B, 81 060411(R) (2010). 